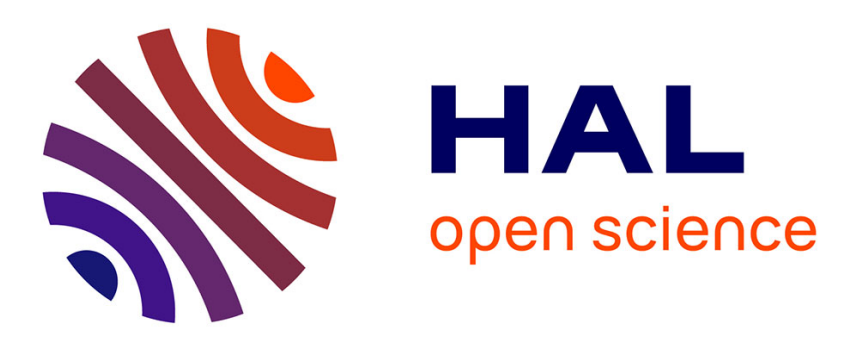

\title{
Nouvelle imagerie dans le lymphœdème : lympho-IRM pour quoi faire ?
}

L. Arrivé

\section{To cite this version:}

L. Arrivé. Nouvelle imagerie dans le lymphœdème : lympho-IRM pour quoi faire ?. JMV-Journal de Médecine Vasculaire, 2018, 43 (2), pp.109. 10.1016/j.jdmv.2017.12.079 hal-01744465

\section{HAL Id: hal-01744465 \\ https://hal.sorbonne-universite.fr/hal-01744465}

Submitted on 27 Mar 2018

HAL is a multi-disciplinary open access archive for the deposit and dissemination of scientific research documents, whether they are published or not. The documents may come from teaching and research institutions in France or abroad, or from public or private research centers.
L'archive ouverte pluridisciplinaire HAL, est destinée au dépôt et à la diffusion de documents scientifiques de niveau recherche, publiés ou non, émanant des établissements d'enseignement et de recherche français ou étrangers, des laboratoires publics ou privés. 


\section{College francais pathologie vasculaire 22032018}

\section{5 minutes plus cinq minutes discussion}

Nouvelles imagerie dans le lymphoedème : lympho-IRM pour quoi faire ?

\section{Arrivé}

Service de Radiologie - Hôpital Saint-Antoine - Assistance publique - Hôpitaux de Paris, 184, rue du Faubourg-Saint-Antoine, 75012 Paris

Faculté de Médecine Pierre et Marie Curie, Université Paris V

(lionel.arrive@aphp.fr)

Mots clés :

Lymphoedème

Lymphographie par résonance magnétique

L'imagerie par résonance magnétique (IRM) est caractérisée par un contraste spontané de très bonne qualité. Il s'agit ici de se servir du contraste spontané de l'imagerie par résonance magnétique et notamment de son excellente capacité à différencier les structures contenant du liquide des tissus solides. Il est possible aujourd'hui d'obtenir en quelques secondes une imagerie bidimensionnelle (2D) de qualité et en quelques minutes une imagerie tridimensionnelle d'excellente qualité (3D). On peut également programmer des séquences spécifiques dont l'objectif est d'extraire uniquement le signal des structures contenant un fluide statique ou à circulation lente à l'exception des autres tissus de l'organisme.

Le principe de ces séquences est de se servir de séquences très pondérées en T2 afin que seules les structures contenant un liquide stationnaire ou un liquide à circulation lente gardent un signal par rapport aux autres tissus de l'organisme. En se servant de séquences natives très fines, il est possible d'obtenir une acquisition 3D qui permet l'analyse de l'ensemble du volume mais également de réaliser des reconstructions dans les trois plans de l'espace en se servant d'algorithme de reconstruction tel que l'algorithme MIP (Maximum Intensity Projection). Avec l'amélioration de ces techniques, il est devenu possible d'analyser des structures canalaires de plus petites tailles et donc des structures lymphatiques canalaires et ganglionnaires.

En pratique courante, nous nous servons d'une séquence permettant une acquisition tridimensionnelle, en respiration libre sans aucune injection de produit de contraste avec une épaisseur de coupe millimétrique ou inframillimétrique et une matrice de $256^{2}$ voire $512^{2}$. Dans ces conditions les dimensions du voxel sont inférieures au millimètre. La séquence est couramment acquise en 3 à 4 minutes en respiration libre en se servant d'une synchronisation à la respiration Dans ces conditions, on visualise toujours très correctement les lymphonoeuds, les canaux lymphatiques inguinaux, iliaques, rétropéritonéaux et le canal thoracique.

On visualise également généralement les canaux lymphatiques principaux des membres inférieurs. Par contre, les lymphatiques normaux du membre supérieur sont beaucoup plus difficiles à voir. La lymphographie par résonance magnétique permet d'effectuer un diagnostic positif du lymphoedème qu'il s'agisse d'un lymphoedème primitif ou d'un lymphoedème secondaire. La lymphographie par résonance magnétique permet d'effectuer une évaluation objective de la gravité, mais elle permet principalement de classer les lymphoedèmes primitifs en variétés aplasique, hypoplasique ou dysplasique en distinguant les formes limitées au réseau canalaire et les formes intéressant les ganglions lymphatiques. La lymphographie peut également être utilisée comme un moyen de surveillance objectif après traitement.

Pas de conflit d'intérêt. 
